\title{
A Moments and Strike Matching Binomial Algorithm for Pricing American Put Options
}

Benjamin Jourdain and Antonino Zanette

\section{$\mathbf{N}^{\circ} 5569$}

May, 82005

Thème NUM 



\title{
A Moments and Strike Matching Binomial Algorithm for Pricing American Put Options
}

\author{
Benjamin Jourdain* and Antonino Zanette ${ }^{\dagger}$ \\ Thème NUM - Systèmes numériques \\ Projet MATHFI \\ Rapport de recherche $\mathrm{n}^{\circ} 5569$ - May, 82005 - 15 pages
}

\begin{abstract}
This paper is dedicated to a new binomial lattice method (MSM) consistent with the Black-Scholes model in the limit of an infinite step number and such that the Strike $K$ is equal to one of the final nodes of the tree. The method is very easy to implement, since the parameters are explicitly given. Asymptotic expansions are obtained for the MSM European Put price and delta, which motivates the use of Richardson extrapolation. A numerical comparison with the best lattice based numerical methods known in literature, shows the efficiency of the proposed algorithm for pricing and hedging American Put options.
\end{abstract}

Key-words: option pricing, hedging, American Put options, tree methods.

\footnotetext{
* CERMICS and Mathfi Project, École Nationale des Ponts et Chaussées, 6 et 8 avenue Blaise Pascal, Cité Descartes, Champs-sur-Marne, 77455 Marne la Vallée cedex 2, France, email: jourdain@cermics.enpc.fr

$\dagger$ Dipartimento di Finanza dell'Impresa e dei Mercati Finanziaria, Universitá di Udine, via Tomadini 30/A, Udine, Italy, email: antonino.zanette@uniud.it
} 


\section{Arbre binômial centré sur le Strike pour le calcul du put américain}

Résumé : Pour un Put de prix d'exercice $K$, nous expliquons comment construire un modèle binômial qui converge vers le modèle de Black-Scholes lorsque le nombre $N$ de pas de temps tend vers l'infini et tel que $K$ coïncide avec une feuille de l'arbre. Nous obtenons des formules explicites qui permettent d'implémenter très facilement cet arbre binômial. Dans le cas d'une option européenne, nous obtenons des développements asymptotiques du prix et du delta calculés sur l'arbre en fonction de $N$, justifiant ainsi l'utilisation de la méthode d'extrapolation de Richardson. Dans le cas américain, nous présentons une comparaison numérique approfondie qui montre l'efficacité de notre approche par rapport aux meilleures méthodes d'arbres proposées dans la littérature.

Mots-clés : évaluation d'options, couverture, put américain, méthodes d'arbres 


\section{Introduction}

Over the last years, significant progress has been made in understanding the convergence behavior of tree methods for pricing and hedging options (see [10], [11], [13], [4] and the references therein). As suggested by Figlewski and Gao [5], there are two sources of error in lattice models for Call or Put options : the first one derives from the approximation of a continuous distribution by a discrete one while the second one comes from the interplay between the Strike and the grid nodes at the final time step. We refer to Gaudenzi, Pressacco, Zanette, Ziani [7] for a detailed analysis of these sources of error and for a recent review, including the evaluation of the speed/precision efficiency of lattice methods (trees, finite differences). With marked differences in the respective technicalities, several lattice methods [2] [5] [6] try to deal with the second source of error in the standard Cox-Rubinstein model (where the up factor $u$ is equal to the inverse of the down factor $d$ ).

The Adaptive Mesh Model (AMM) introduced by Figlewski and Gao [5] resorts to refining the grid around the strike and at maturity at the cost of very little additional computation time.

The BBS method introduced by Broadie and Detemple [2] replaces at any node of the last but one time before maturity, the binomial continuation value with the Black-Scholes European one [1]. A two-points Richardson extrapolation aimed at improving the convergence leads to the BBSR method. The BI(R) method, that is Binomial Interpolated (with Richardson extrapolation), introduced by Gaudenzi and Pressacco [6] tries to recover the regularity of the sequences giving the Cox-Rubinstein price of the European at the money options respectively for even and for odd step numbers (see [4] and Remark 1 below). For even step numbers, this regularity comes from the coincidence of the Strike with a final node of the Cox-Rubinstein tree. The logic of the BI approach then is to create a set of computational options, each one with a computational Strike lying exactly on a final node of the tree. The value of the option with the contractual Strike is then obtained by interpolation of the values of the computational options. Furthermore, it is possible to exploit the recovered regularity using a two-points Richardson extrapolation : this leads to the BIR method.

In the present paper, we investigate binomial trees with parameters up factor $u$, down factor $d$ and up probability $p_{u}$ such that the contractual Strike itself coincides with a final node of the tree. In order to obtain a tree consistent with the Black-Scholes model in the limit of an infinite step number, we have to check two moments matching conditions. Of course, since we are provided with three equations with three unknowns, we cannot impose $u=1 / d$ like in the standard Cox-Rubinstein model. The solution of the system with three equations is easily explicited and we name the corresponding tree MSM (Moments and Strike Matching).

The paper is organized as follows: in section 1, we introduce the classical Cox-Ross-Rubinstein binomial approximation of the Black-Scholes model. Section 2 is devoted to the construction of MSM trees. In Section 3, we give asymptotic results for the MSM Put price and delta in the European case which justify the use of Richardson extrapolation (MSMR method). In section 4, we present the MSMR method for American Put options. Finally, in section 5, a comparison with the best lattice based numerical methods known in literature is offered using a large and reliable random sample of 5.000 options.

\section{The binomial approximation of the Black-Scholes model}

In this paper, we consider a market model where the evolution of a risky asset is governed by the Black-Scholes stochastic differential equation

$$
\frac{d S_{t}}{S_{t}}=r d t+\sigma d W_{t}, \quad S_{0}=s_{0}
$$


in which, under the so called risk neutral probability measure, $\left(W_{t}\right)_{0 \leq t \leq T}$ is a standard Brownian motion. The nonnegative constant $r$ is the interest rate and the positive constant $\sigma$ is the volatility of the risky asset.

The value at time $t=0$ of a plain vanilla American Put option on the risky underlying with maturity $T$, is, in the connection with Optimal Stopping Theory, given by:

$$
v\left(0, s_{0}\right)=\sup _{\tau \in \mathcal{T}_{0, T}} E\left(e^{-r \tau}\left(K-S_{\tau}\right)^{+}\right)
$$

where $\mathcal{T}_{0, T}$ is the set of all stopping times with values in $[0, T]$.

Unlike European options, American Put options cannot be valued by closed-form formulae and require the use of approximation methods (see for example [8]). We are concerned with discrete numerical methods and recall that there are two main discrete numerical approaches: one is the probabilistic approach based on the approximation of diffusion by Markov chains (tree methods), the other one is the analytic approach related to the discretization of the variational inequality satisfied by the option value (finite difference or finite elements methods). The multiplicative binomial Cox-Ross-Rubinstein model [3] has an interest on his own as a basic discrete-time model for the underlying asset of a financial derivative, converging to the diffusion process (1) under appropriate conditions. One of its main attractive feature is the easiness of standard option pricing by backward induction.

Let $N$ denote the step number of the tree and $\Delta T=\frac{T}{N}$ the corresponding time-step. The lognormal diffusion process $\left(S_{n \Delta T}\right)_{0 \leq n \leq N}$ is approximated by the Cox-Ross-Rubinstein binomial process $\left(s_{0} \prod_{j=1}^{n} Y_{j}\right)_{0 \leq n \leq N}$ where the random variables $Y_{1}, \ldots, Y_{N}$ are independent and identically distributed with values in $\{d, u\}$. Let us denote by $p_{u}=\mathbb{P}\left(Y_{n}=u\right)=1-\mathbb{P}\left(Y_{n}=d\right)$. Kushner's theorem [9] says that the local consistency conditions, that is the matching at the first order of the first and second moments of the logarithmic increments of the approximating chain with those of the continuous-time limit grant the convergence of the expectations of smooth functionals. These first two moments matching conditions read

$$
\left\{\begin{array}{l}
p_{u} \log u+\left(1-p_{u}\right) \log d=\left(r-\frac{1}{2} \sigma^{2}\right) \Delta T \\
p_{u}(\log u)^{2}+\left(1-p_{u}\right)(\log d)^{2}=\sigma^{2} \Delta T
\end{array}\right.
$$

In the Cox-Ross-Rubinstein model, the price at time $n \in\{0, \ldots, N\}$ of the European (resp. American) Put option is given by $v\left(n, s_{0} \prod_{j=1}^{n} Y_{j}\right)$ where the functions $v(n, x)$ can be computed by the following backward dynamic programming equations

$$
\left\{\begin{array}{l}
v_{N}(N, x)=(K-x)^{+} \\
v_{N}(n, x)=\max \left(\psi(x), \frac{1}{u p_{u}+d\left(1-p_{u}\right)}\left[p_{u} v_{N}(n+1, x u)+\left(1-p_{u}\right) v_{N}(n+1, x d)\right]\right),
\end{array}\right.
$$

where $\psi \equiv 0\left(\right.$ resp. $\left.\psi(x)=(K-x)^{+}\right)$. In the European case,

$$
v_{N}(n, x)=\frac{1}{\left(u p_{u}+d\left(1-p_{u}\right)\right)^{N-n}} \mathbb{E}\left[\left(K-x \prod_{j=n+1}^{N} Y_{j}\right)^{+}\right] .
$$

The presence of $\mathbb{E}\left[Y_{1}\right]=u p_{u}+d\left(1-p_{u}\right)$ in the actualization factors in (3) and (4) ensures that the prices computed in the binomial model are arbitrage-free. In order to obtain explicit expressions for the parameters of MSM trees, we prefer to impose the first equality in (2) rather than the more usual condition $u p_{u}+d\left(1-p_{u}\right)=e^{r \Delta t}$. Notice that under $(2)$, the last equality holds up to a term with order $\mathcal{O}\left((\Delta T)^{2}\right)$.

The initial price (resp. delta) of the Put option in the Black-Scholes model can be approximated by 
$v_{N}\left(0, s_{0}\right)$ (resp. the amount $\frac{v_{N}\left(1, s_{0} u\right)-v_{N}\left(1, s_{0} d\right)}{s_{0}(u-d)}$ of risky asset in the replication portfolio on the first time-step in the Cox-Ross-Rubinstein model). Notice that in order to obtain the approximate price and delta, one only needs to compute $\left(v_{N}\left(n, s_{0} u^{k} d^{n-k}\right), 0 \leq k \leq n\right)$ by backward induction on $n$.

The usual Cox-Ross-Rubinstein tree (henceforth denoted CRR) corresponds to the choice $u=\frac{1}{d}=$ $e^{\sigma \sqrt{\Delta T}}$ and $d+(u-d) p_{u}=e^{r \Delta T}$. This leads to $p_{u}=\frac{e^{r \Delta T}-e^{-\sigma \sqrt{\Delta T}}}{e^{\sigma \sqrt{\Delta T}}-e^{-\sigma \sqrt{\Delta T}}}$. When $\Delta T$ is small enough i.e. when $N$ is large enough, the above value of $p_{u}$ belongs to ]0,1[. For this choice of $u, d$ and $p_{u}$, the second equality in (2) is satisfied whereas the difference between both sides of the first equality in (2) is of order $(\Delta T)^{2}$. This is sufficient to ensure convergence to the Black-Scholes model in the limit $N \rightarrow+\infty$. Let $P_{N}^{\mathrm{CRR}}$ and $P_{\mathrm{BS}}$ denote the initial price of the European Put option with maturity $T$ and strike $K$ respectively in the Cox-Ross-Rubinstein model and in the Black-Scholes model. Using the Call-Put parity relations in both models and results given for the Call option in [4], one gets

$$
P_{N}^{\mathrm{CRR}}=P_{\mathrm{BS}}-\frac{K e^{-r T}}{N} e^{-\frac{d_{2}^{2}}{2}} \sqrt{\frac{2}{\pi}}\left[\kappa_{N}\left(\kappa_{N}-1\right) \sigma \sqrt{T}+D_{1}\right]+\mathcal{O}\left(\frac{1}{N^{3 / 2}}\right),
$$

where $d_{2}=\frac{\log \left(\frac{s_{0}}{K}\right)+\left(r-\frac{\sigma^{2}}{2}\right) T}{\sigma \sqrt{T}}, \kappa_{N}$ denotes the fractional part of $\frac{\log \left(\frac{K}{s_{0}}\right)}{2 \sigma} \sqrt{\frac{N}{T}}-\frac{N}{2}$ and $D_{1}$ is a constant. Because the sequence $\left(\kappa_{N}\right)_{N}$ is oscillating although bounded, in the approximation $2 P_{2 N}^{\mathrm{CRR}}-P_{N}^{\mathrm{CRR}}$ of $P_{\mathrm{BS}}$ obtained using Richardson extrapolation, the term with order $1 / N$ does not vanish which explains the poor numerical behaviour of this approximation. If the Strike $K$ is equal to one of the final nodes $\left(s_{0} e^{(2 k-N) \sigma \sqrt{\Delta T}}\right)_{0 \leq k \leq N}$ of the tree, then one has $\kappa_{N}=0$. This justifies our interest in trees such that the Strike coincides with one of the final nodes.

Remark 1 Suppose that the Put option is at the money i.e. that $K=s_{0}$. Then for $N=2 m$ even, the Strike $K$ coincides with the $(m+1)$-th final node of the $(C R R)$ tree, and one has

$$
P_{2 m}^{\mathrm{CRR}}=P_{\mathrm{BS}}-\frac{D_{1} K e^{-r T}}{2 m} e^{-\frac{d_{2}^{2}}{2}} \sqrt{\frac{2}{\pi}}+\mathcal{O}\left(\frac{1}{m^{3 / 2}}\right)
$$

As a consequence the rate of convergence of $2 P_{4 m}^{\mathrm{CRR}}-P_{2 m}^{\mathrm{CRR}}$ to $P_{\mathrm{BS}}$ is $\mathcal{O}\left(\frac{1}{m^{3 / 2}}\right)$. For $N=2 m+1$ odd, one has $\kappa_{N}=1 / 2$ which ensures that

$$
P_{2 m+1}^{\mathrm{CRR}}=P_{\mathrm{BS}}-\frac{K e^{-r T}}{2 m} e^{-\frac{d_{2}^{2}}{2}} \sqrt{\frac{2}{\pi}}\left[D_{1}-\frac{\sigma \sqrt{\Delta T}}{4}\right]+\mathcal{O}\left(\frac{1}{m^{3 / 2}}\right) .
$$

and the rate of convergence of $2 P_{4 m+1}^{\mathrm{CRR}}-P_{2 m+1}^{\mathrm{CRR}}$ to $P^{\mathrm{BS}}$ is $\mathcal{O}\left(\frac{1}{m^{3 / 2}}\right)$.

Remark 2 According to our numerical experiments, the expression of $D_{1}$ given in Corollary 4.1 [4] is not correct. It seems to us that in the expansions $\frac{1}{2}\left(1+\left(\frac{r}{\sigma}-\varepsilon \frac{\sigma}{2}\right) \sqrt{\frac{T}{N}}+\left(\varepsilon \frac{r^{2}}{2 \sigma}-\frac{r \sigma}{6}+\varepsilon \frac{\sigma^{3}}{24}\right)\left(\frac{T}{N}\right)^{3 / 2}\right)+$ $\mathcal{O}\left(\frac{1}{N^{2}}\right)$ valid for $p_{u}=\frac{e^{r \Delta T}-e^{-\sigma \sqrt{\Delta T}}}{e^{\sigma \sqrt{\Delta T}}-e^{-\sigma \sqrt{\Delta T}}}$ with $\varepsilon=1$ and $\frac{u p_{u}}{\left(d+(u-d) p_{u}\right)}=\frac{e^{\sigma \sqrt{\Delta T}}-e^{-r \Delta T}}{e^{\sigma \sqrt{\Delta T}}-e^{-\sigma \sqrt{\Delta T}}}$ with $\varepsilon=-1$, it would be necessary to take into account not only the term with order $1 / \sqrt{N}$ but also the term with order $1 / N^{3 / 2}$ to obtain the correct constant (see Lemma 2 below). 


\section{Construction of MSM trees}

Instead of requiring $u=\frac{1}{d}$ as in the Cox-Ross-Rubinstein model, we propose in the MSM method to ensure that the Strike $K$ is the $(k+1)$-th (with $k \in\{1, \ldots, N-1\}$ ) final node of the tree : $K=s_{0} u^{k} d^{N-k}$ which also writes $\frac{1}{N} \log \left(\frac{K}{s_{0}}\right)=q \log u+(1-q) \log d$ where $q=\frac{k}{N}$.

Complementing this equation with the first two moment matching conditions (2), we want to find $\left(\log u, \log d, p_{u}\right)$ with $\log u>\log d$ and $\left.p_{u} \in\right] 0,1[$ solving the following system of equations with unknowns $(x, y, p)$

$$
\left\{\begin{array}{l}
q x+(1-q) y=\alpha \\
p x+(1-p) y=\beta \\
p x^{2}+(1-p) y^{2}=\gamma
\end{array}\right.
$$

where $\alpha=\frac{1}{N} \log \left(\frac{K}{s_{0}}\right), \beta=\left(r-\frac{\sigma^{2}}{2}\right) \Delta T$ and $\gamma=\sigma^{2} \Delta T$. The coefficients $(q, \alpha, \beta, \gamma)$ belong to $] 0,1\left[\times \mathbb{R}^{2} \times\right.$ $\mathbb{R}_{+}^{*}$.

Because of the strict convexity of the square function, the two last equations imply that $\gamma>\beta^{2}$ is a necessary condition for the existence of a solution. This condition obviously holds if $N$ is large enough. It turns out to be sufficient :

Lemma 1 Assume that $\gamma>\beta^{2}$ and $\left.q \in\right] 0,1[$. Then the system of equations (5) admits exactly two solutions $\left(p_{i}, x_{i}, y_{i}\right)_{i \in\{1,2\}}$ in $] 0,1\left[\times\left\{(x, y) \in \mathbb{R}^{2}: x \neq y\right\}\right.$. These solutions are such that $\left(x_{1}-y_{1}\right)\left(x_{2}-\right.$ $\left.y_{2}\right)<0$ and by convention, we choose the indexes so that $x_{1}>y_{1}$ and $x_{2}<y_{2}$.

Remark 3 It is easy to check that the solutions $\left(\bar{p}_{i}, \bar{x}_{i}, \bar{y}_{i}\right)_{i \in\{1,2\}}$ of the system obtained by replacing $q$ by $\bar{q}=1-q$ in (5) are given by $\left(\bar{p}_{i}, \bar{x}_{i}, \bar{y}_{i}\right)=\left(1-p_{3-i}, y_{3-i}, x_{3-i}\right)$ for $i \in\{1,2\}$ where $\left(p_{i}, x_{i}, y_{i}\right)_{i \in\{1,2\}}$ denote the solutions of the original system. In particular, when $q=\frac{1}{2}$, the two solutions of (5) are linked by $\left(p_{2}, x_{2}, y_{2}\right)=\left(1-p_{1}, y_{1}, x_{1}\right)$.

Proof : We assume that $\gamma>\beta^{2}$ and $\left.q \in\right] 0,1[$. We are first going to deal with the case $\alpha \neq \beta$ before treating the case $\alpha=\beta$.

Case $\alpha \neq \beta$ : Then the first two equations are equivalent to $p \neq q, x=\alpha+(1-q) \frac{\beta-\alpha}{p-q}$ and $y=\alpha-q \frac{\beta-\alpha}{p-q}$. Replacing these expressions of $x$ end $y$ in the last equation, we deduce that the system (5) is equivalent to

$$
\left\{\begin{array} { l } 
{ p \neq q } \\
{ x = \alpha + ( 1 - q ) \frac { \beta - \alpha } { p - q } , y = \alpha - q \frac { \beta - \alpha } { p - q } } \\
{ a p ^ { 2 } + b p + c = 0 }
\end{array} \quad \text { where } \left\{\begin{array}{l}
a=(\alpha-\beta)^{2}+\left(\gamma-\beta^{2}\right) \\
b=-(\alpha-\beta)^{2}-2 q\left(\gamma-\beta^{2}\right) \\
c=q^{2}\left(\gamma-\beta^{2}\right)
\end{array}\right.\right.
$$

The discriminant $\Delta=(\alpha-\beta)^{2}\left[(\alpha-\beta)^{2}+4 q(1-q)\left(\gamma-\beta^{2}\right)\right]$ of the last equation is positive and we deduce that this equation admits two roots :

$p_{1}=\frac{-b+(\beta-\alpha) \sqrt{(\alpha-\beta)^{2}+4 q(1-q)\left(\gamma-\beta^{2}\right)}}{2 a}$ and $\quad p_{2}=\frac{-b+(\alpha-\beta) \sqrt{(\alpha-\beta)^{2}+4 q(1-q)\left(\gamma-\beta^{2}\right)}}{2 a}$.

Since the polynomial $P(z)=a z^{2}+b z+c$ also writes $P(z)=(\alpha-\beta)^{2}\left(z^{2}-z\right)+\left(\gamma-\beta^{2}\right)(z-q)^{2}$, one easily checks that $P(z)>0$ for $z \in \mathbb{R} \backslash] 0,1[$ and that $P(q)<0$. Therefore one of the roots belongs to 
] $0, q[$ and the other one to $] q, 1\left[\right.$. More precisely $\operatorname{sign}\left(p_{1}-q\right)=\operatorname{sign}(\beta-\alpha)=\operatorname{sign}\left(q-p_{2}\right)$. Hence the system (5) has exactly two solutions $\left(p_{1}, x_{1}, y_{1}\right)$ and $\left(p_{2}, x_{2}, y_{2}\right)$ in $] 0,1\left[\times\left\{(x, y) \in \mathbb{R}^{2}: x \neq y\right\}\right.$ with

$$
x_{i}=\alpha+(1-q) \frac{\beta-\alpha}{p_{i}-q} \text { and } y_{i}=\alpha-q \frac{\beta-\alpha}{p_{i}-q} \text { for } i \in\{1,2\} .
$$

Notice that $x_{i}=y_{i}+\frac{\beta-\alpha}{p_{i}-q}$, which ensures that $x_{1}>y_{1}$ and $x_{2}<y_{2}$.

Case $\alpha=\beta$ : Since we are looking for solutions with $x \neq y$, the first two equations in the system (5) are equivalent to $p=q$ and $x=(\beta+(q-1) y) / q$. Replacing these expressions of $p$ and $x$ in the last equation, we deduce that (5) is equivalent to

$$
\left\{\begin{array}{l}
p=q \\
x=(\beta+(q-1) y) / q \\
y^{2}-2 \beta y+\frac{\beta^{2}-q \gamma}{1-q}=0 .
\end{array}\right.
$$

The discriminant of the last equation $\Delta=\frac{4 q\left(\gamma-\beta^{2}\right)}{1-q}$ is positive and this equation has two solutions

$$
y_{1}=\beta-\sqrt{\frac{q\left(\gamma-\beta^{2}\right)}{1-q}} \text { and } y_{2}=\beta+\sqrt{\frac{q\left(\gamma-\beta^{2}\right)}{1-q}} .
$$

The corresponding values for $x$

$$
x_{1}=\beta+\sqrt{\frac{(1-q)\left(\gamma-\beta^{2}\right)}{q}} \text { and } x_{2}=\beta-\sqrt{\frac{(1-q)\left(\gamma-\beta^{2}\right)}{q}},
$$

obviously satisfy $x_{1}>y_{1}$ and $x_{2}<y_{2}$. Hence the system (5) has exactly two solutions $\left(q, x_{1}, y_{1}\right)$ and $\left(q, x_{2}, y_{2}\right)$ in $] 0,1\left[\times\left\{(x, y) \in \mathbb{R}^{2}: x \neq y\right\}\right.$.

Let us turn back to the construction of MSM trees. Assuming that $\gamma>\beta^{2}$, for $q=k / N$ with $k \in\{1, \ldots, N-1\}$, the solutions $\left(p_{i}, x_{i}, y_{i}\right)_{i \in\{1,2\}}$ of (5) given by Lemma 1 provide two trees :

- the first one with $p_{u}=p_{1}, \log u=x_{1}$ and $\log d=y_{1}$ is such that the $(k+1)$-th final node $s_{0} u^{k} d^{N-k}$ of the tree is equal to the Strike $K$,

- the second one with $p_{u}=1-p_{2}, \log u=y_{2}$ and $\log d=x_{2}$ is such that the $(N-k)$-th final node $s_{0} u^{N-k} d^{k}$ of the tree is equal to the Strike $K$.

Notice that according to Remark 3, solving system (5) for $q=(N-k) / N$ leads to the two same trees. And when $N$ is even and $k=N / 2$, both trees are equal.

Corollary 1 Assume that $N \geq\left(\frac{r}{\sigma}-\frac{\sigma}{2}\right)^{2} T$. Then for any $k \in\{1, \ldots, N-1\}$, there is a unique MSM tree with $N$ steps and parameters $\left(p_{u}, \log u, \log d\right)$ (with $\left.p_{u} \in\right] 0,1[$ and $\log u>\log d$ ) satisfying the two first moment matching conditions (2) and such that the strike $K$ is equal to the $(k+1)$-th final node of the tree : $K=s_{0} u^{k} d^{N-k}$.

The parameters of this tree are given by

$$
\left\{\begin{array} { l } 
{ p _ { u } = \frac { ( \alpha - \beta ) ^ { 2 } + 2 q ( \gamma - \beta ^ { 2 } ) - ( \alpha - \beta ) \sqrt { ( \alpha - \beta ) ^ { 2 } + 4 q ( 1 - q ) ( \gamma - \beta ^ { 2 } ) } } { 2 ( ( \alpha - \beta ) ^ { 2 } + ( \gamma - \beta ^ { 2 } ) ) } } \\
{ \operatorname { l o g } u = \alpha + ( 1 - q ) \frac { \beta - \alpha } { p _ { u } - q } }
\end{array} \quad \text { where } \begin{array} { l } 
{ q = \frac { k } { N } } \\
{ \operatorname { l o g } d = \alpha - q \frac { \beta - \alpha } { p _ { u } - q } }
\end{array} \quad \left\{\begin{array}{l}
N \log \left(\frac{K}{s_{0}}\right) \\
\beta=\left(r-\frac{\sigma^{2}}{2}\right) \frac{T}{N} \\
\text { with convention } \frac{\beta-\alpha}{p_{u}-q}=\sqrt{\frac{\gamma-\beta^{2}}{q(1-q)}} \text { when } \alpha=\beta
\end{array} .\right.\right.
$$


Remark 4 - Notice that in the above equations for $\log u$ and $\log d$, the conventional value of $\frac{\beta-\alpha}{p_{u}-q}$ when $\alpha=\beta$ is $\sqrt{\frac{\gamma-\beta^{2}}{q(1-q)}}=\lim _{\substack{\alpha \neq \beta \\ \alpha \rightarrow \beta}} \frac{\beta-\alpha}{p_{u}-q}$.

- For any $k \in\{1, \ldots, N-1\}$ the $M S M$ tree such that $K=s_{0} u^{k} d^{N-k}$ is recombining since $u$ and $d$ remain constant within the tree. But unless $K=s_{0} e^{(2 k-N) \sigma \sqrt{\Delta t}}$, because the standard CRR tree is the only tree such that $u=\frac{1}{d}$ and the second moment matching condition in (2) is satisfied, the MSM tree is not symmetric in the log scale : $\log u \neq-\log d$. Then, unlike in the standard CRR tree, the nodes at time $n$ are not a subset of the nodes at time $n+2$.

\section{Asymptotics of the European Put price and delta in MSM trees}

Because of Remark 1, we may expect that the initial price $P_{2 m}$ of the European Put option with strike $K$ and maturity $T$ in the MSM model with $N=2 m$ even and $k=m$ is such that $P_{2 m}=$ $P_{\mathrm{BS}}+\frac{C}{m}+\mathcal{O}\left(\frac{1}{m^{3 / 2}}\right)$ with $C$ not depending on $m$ ( $P_{\mathrm{BS}}$ denotes the initial Black-Scholes price of the Put option). Following the ideas of [4], we are going to obtain such an expansion. By (4) and since $K=s_{0} u^{m} d^{m}$, one has

$P_{2 m}=\frac{K}{\left(d+(u-d) p_{u}\right)^{2 m}} \sum_{j=0}^{m-1}\left(\begin{array}{c}2 m \\ j\end{array}\right) p_{u}^{j}\left(1-p_{u}\right)^{2 m-j}-s_{0} \sum_{j=0}^{m-1}\left(\begin{array}{c}2 m \\ j\end{array}\right)\left(\frac{u p_{u}}{d+(u-d) p_{u}}\right)^{j}\left(\frac{d\left(1-p_{u}\right)}{d+(u-d) p_{u}}\right)^{2 m-j}$

and for $y \in\{0,1\}, v_{2 m}\left(1, s_{0} u^{y} d^{1-y}\right)=\frac{K}{\left(d+(u-d) p_{u}\right)^{2 m-1}} \sum_{j=0}^{m-1}\left(\begin{array}{c}2 m-1 \\ j\end{array}\right) p_{u}^{j}\left(1-p_{u}\right)^{2 m-1-j}$

$$
-s_{0} u^{y} d^{1-y} \sum_{j=0}^{m-1}\left(\begin{array}{c}
2 m-1 \\
j
\end{array}\right)\left(\frac{u p_{u}}{d+(u-d) p_{u}}\right)^{j}\left(\frac{d\left(1-p_{u}\right)}{d+(u-d) p_{u}}\right)^{2 m-1-j} .
$$

One deduces that the initial delta $\delta_{2 m}=\frac{v_{2 m}\left(0, s_{0} u\right)-v_{2 m}\left(0, s_{0} d\right)}{s_{0}(u-d)}$ of the European Put in the MSM tree with $2 m$ steps and $k=m$ is given by

$$
\delta_{2 m}=-\sum_{j=0}^{m-1}\left(\begin{array}{c}
2 m-1 \\
j
\end{array}\right)\left(\frac{u p_{u}}{d+(u-d) p_{u}}\right)^{j}\left(\frac{d\left(1-p_{u}\right)}{d+(u-d) p_{u}}\right)^{2 m-1-j}
$$

In the above formulas, $u, d$ and $p_{u}$ depend on $m$. To obtain the asymptotic expansion of $P_{2 m}$ and $\delta_{2 m}$ in powers of $1 / \sqrt{m}$, we are going to compute the asymptotic expansions of these coefficients. More precisely, we need the asymptotic expansions of $p_{u}, \frac{u p_{u}}{d+(u-d) p_{u}}$ and $\left(d+(u-d) p_{u}\right)^{-2 m}$ :

Lemma 2 For the $M S M$ tree with $N=2 m$ steps and $k=m$, one has

$$
\left\{\begin{array} { l } 
{ p _ { u } = \frac { 1 } { 2 } + \frac { d _ { 2 } } { 2 \sqrt { 2 m } } + \frac { \mu } { ( 2 m ) ^ { 3 / 2 } } + \mathcal { O } ( \frac { 1 } { m ^ { 5 / 2 } } ) } \\
{ \frac { u p _ { u } } { d + ( u - d ) p _ { u } } = \frac { 1 } { 2 } + \frac { d _ { 1 } } { 2 \sqrt { 2 m } } + \frac { \nu } { ( 2 m ) ^ { 3 / 2 } } + \mathcal { O } ( \frac { 1 } { m ^ { 2 } } ) } \\
{ ( d + ( u - d ) p _ { u } ) ^ { - 2 m } = e ^ { - r T } ( 1 + \frac { \eta } { m } ) + \mathcal { O } ( \frac { 1 } { m ^ { 3 / 2 } } ) }
\end{array} \quad \text { where } \quad \left\{\begin{array}{l}
d_{1}=\frac{\log \left(\frac{s_{0}}{K}\right)+\left(r+\frac{\sigma^{2}}{2}\right) T}{\sigma \sqrt{T}} \\
d_{2}=d_{1}-\sigma \sqrt{T}
\end{array}\right.\right.
$$

and $\mu=\frac{d_{2} \log \left(\frac{s_{0}}{K}\right)\left[\log \left(\frac{K}{s_{0}}\right)+\left(\sigma^{2}-2 r\right) T\right]}{4 \sigma^{2} T}, \nu=\frac{d_{1} \log \left(\frac{s_{0}}{K}\right)\left[\log \left(\frac{K}{s_{0}}\right)+\left(\sigma^{2}-2 r\right) T\right]}{4 \sigma^{2} T}-\frac{\left(r-\frac{\sigma}{2}\right)^{2} T^{3 / 2}}{2 \sigma}-\frac{\sigma^{2} T d_{2}}{2}-\frac{\sigma^{3} T^{3 / 2}}{6}$ and $\eta=\frac{1}{4}\left(r-\frac{\sigma^{2}}{2}\right)^{2} T^{2}+\frac{1}{24} \sigma^{4} T^{2}+\frac{d_{2}}{6} \sigma^{3} T^{3 / 2}$. 
We remark that the expansions of both $p_{u}$ and $\frac{u p_{u}}{d+(u-d) p_{u}}$ write $\frac{1}{2}+\frac{a}{\sqrt{2 m}}+\frac{b}{(2 m)^{3 / 2}}+\mathcal{O}\left(\frac{1}{m^{2}}\right)$. This justifies our interest in the next Lemma which is obtained following the approach developped in [4]:

Lemma 3 If $p=\frac{1}{2}+\frac{\tilde{p}}{\sqrt{2 m}}+\frac{\tilde{\tilde{p}}}{(2 m)^{3 / 2}}+o\left(\frac{1}{m^{3 / 2}}\right)$ in the limit $m \rightarrow+\infty$, then

$$
\begin{aligned}
& \sum_{j=0}^{m-1}\left(\begin{array}{c}
2 m \\
j
\end{array}\right) p^{j}(1-p)^{2 m-j}=\mathcal{N}(-2 \tilde{p})-e^{-2 \tilde{p}^{2}}\left(\frac{1}{\sqrt{4 \pi m}}+\frac{1}{m \sqrt{2 \pi}}\left(\tilde{p}^{3}+\tilde{\tilde{p}}-\frac{\tilde{p}}{4}\right)\right)+\mathcal{O}\left(\frac{1}{m^{3 / 2}}\right) \\
& \sum_{j=0}^{m-1}\left(\begin{array}{c}
2 m-1 \\
j
\end{array}\right) p^{j}(1-p)^{2 m-1-j}=\mathcal{N}(-2 \tilde{p})+\frac{e^{-2 \tilde{p}^{2}}}{m \sqrt{2 \pi}}\left(\frac{\tilde{p}}{4}-\tilde{p}^{3}-\tilde{\tilde{p}}\right)+\mathcal{O}\left(\frac{1}{m^{3 / 2}}\right),
\end{aligned}
$$

where $\mathcal{N}(x)=\int_{-\infty}^{x} e^{-y^{2} / 2} \frac{d y}{\sqrt{2 \pi}}$ denotes the cumulative distribution function of the Normal law.

Before proving Lemmas 2 and 3, let us deduce the expansions of $P_{2 m}$ and $\delta_{2 m}$. By (6),

$$
\begin{aligned}
P_{2 m}= & K e^{-r T}\left(1+\frac{\eta}{m}+\mathcal{O}\left(\frac{1}{m^{3 / 2}}\right)\right)\left(\mathcal{N}\left(-d_{2}\right)-e^{-\frac{d_{2}^{2}}{2}}\left(\frac{1}{\sqrt{4 \pi m}}+\frac{1}{m \sqrt{2 \pi}}\left(\frac{d_{2}^{3}-d_{2}}{8}+\mu\right)\right)+o\left(\frac{1}{m}\right)\right) \\
& -s_{0}\left(\mathcal{N}\left(-d_{1}\right)-e^{-\frac{d_{1}^{2}}{2}}\left(\frac{1}{\sqrt{4 \pi m}}+\frac{1}{m \sqrt{2 \pi}}\left(\frac{d_{1}^{3}-d_{1}}{8}+\nu\right)\right)+o\left(\frac{1}{m}\right)\right) .
\end{aligned}
$$

Since $e^{-\frac{d_{1}^{2}}{2}}=e^{-\frac{d_{2}^{2}}{2}} \frac{K e^{-r T}}{s_{0}}$, the terms with order $1 / \sqrt{m}$ cancel.

For the delta, the computations are very easy. As $P_{\mathrm{BS}}=K e^{-r T} \mathcal{N}\left(-d_{2}\right)-s_{0} \mathcal{N}\left(-d_{1}\right)$ and $\delta_{\mathrm{BS}}=$ $-\mathcal{N}\left(-d_{1}\right)$ are respectively the initial Black-Scholes price and delta of the European Put option, we obtain

Corollary 2 As $m$ tends to infinity,

$P_{2 m}=P_{\mathrm{BS}}+\frac{C_{P}}{m}+\mathcal{O}\left(\frac{1}{m^{3 / 2}}\right)$ with $C_{P}=K e^{-r T}\left(\eta \mathcal{N}\left(-d_{2}\right)+\frac{e^{-\frac{d_{2}^{2}}{2}}}{\sqrt{2 \pi}}\left(\frac{d_{1}^{3}+d_{2}-d_{2}^{3}-d_{1}}{8}+\nu-\mu\right)\right)$ $\delta_{2 m}=\delta_{\mathrm{BS}}+\frac{C_{\delta}}{m}+\mathcal{O}\left(\frac{1}{m^{3 / 2}}\right)$ with $C_{\delta}=\frac{e^{-\frac{d_{1}^{2}}{2}}}{\sqrt{2 \pi}}\left(\frac{d_{1}^{3}-d_{1}}{8}+\nu\right)$,

where $d_{1}, d_{2}, \mu, \nu$ and $\eta$ are defined in Lemma 2.

Numerical results in table 1 illustrate that in the above expansions, the next term is a $\mathcal{O}\left(\frac{1}{m^{2}}\right)$ rather than a $\mathcal{O}\left(\frac{1}{m^{3 / 2}}\right)$.

\begin{tabular}{|c|c|c|c|c|}
\hline$m$ & 100 & 200 & 400 & 800 \\
\hline$m^{2} \times\left(P_{2 m}-P_{\mathrm{BS}}-\frac{C_{P}}{m}\right)$ & 0.0139063 & 0.0136744 & 0.0135587 & 0.0135030 \\
\hline$m^{2} \times\left(\delta_{2 m}-\delta_{\mathrm{BS}}-\frac{C_{\delta}}{m}\right)$ & -0.0017242 & -0.0017223 & -0.0017213 & -0.0017209 \\
\hline
\end{tabular}

Table 1: Option parameters $s_{0}=100, K=107.96, T=0.8375, r=0.0107, \sigma=0.2168$

For integers $p$ and $i \in\{1, \ldots, p-1\}$, one could study the asymptotic expansion of the European Put price in the MSM tree with $N=p * q$ steps and $k=i * q$. In table 2, we give numerical results 
for $p=8$, which show that unless $i=p / 2=4$, the convergence rate to the Black-Scholes price is obviously $1 / \sqrt{N}$ or equivalently $1 / \sqrt{q}$. The case $i=4$ illustrates corollary 2 : when the step number is multiplied by 4 , the value in the corresponding column is divided by 2 , which means that the difference between the MSM price and the Black-Scholes price is divided by $2 \times \sqrt{4}=4$.

\begin{tabular}{|r|r|c|c|c|c|c|c|r|}
\hline & $N$ & $i=1$ & $i=2$ & $i=3$ & $\mathbf{i}=\mathbf{4}$ & $i=5$ & $i=6$ & $i=7$ \\
\hline Price & 200 & 1.3297 & 0.6770 & 0.2574 & $\mathbf{- 0 . 1 0 5 1}$ & -0.4878 & -0.9878 & -1.9273 \\
& 800 & 1.4902 & 0.7580 & 0.3162 & $\mathbf{- 0 . 0 5 2 5}$ & -0.4314 & -0.9134 & -1.7892 \\
& 3200 & 1.5677 & 0.7976 & 0.3453 & $\mathbf{- 0 . 0 2 6 2}$ & -0.4029 & -0.8754 & -1.7173 \\
& 12800 & 1.6058 & 0.8173 & 0.3598 & $\mathbf{- 0 . 0 1 3 1}$ & -0.3886 & -0.8561 & -1.6806 \\
\hline Delta & 200 & 0.3466 & 0.1767 & 0.0802 & $\mathbf{0 . 0 0 2 5 7}$ & -0.0746 & -0.1694 & -0.3332 \\
& 800 & 0.3431 & 0.1748 & 0.0787 & $\mathbf{0 . 0 0 1 2 8}$ & -0.0759 & -0.1712 & -0.3364 \\
& 3200 & 0.3414 & 0.1739 & 0.0780 & $\mathbf{0 . 0 0 0 6 4}$ & -0.0766 & -0.1721 & -0.3381 \\
& 12800 & 0.3406 & 0.1734 & 0.0777 & $\mathbf{0 . 0 0 0 3 2}$ & -0.0770 & -0.1725 & -0.3389 \\
\hline
\end{tabular}

Table 2: $\sqrt{N} *(M S M(k=i *(N / 8))-B S)$ (see table 1 for the parameters)

Proof of Lemma 2 : We only give elements of proof in the case $K \neq s_{0} e^{\left(r-\frac{\sigma^{2}}{2}\right) T}$. Computations are similar but easier when $K=s_{0} e^{\left(r-\frac{\sigma^{2}}{2}\right) T}$.

One has $q=m / 2 m=1 / 2, \alpha=\frac{1}{2 m} \log \left(\frac{K}{s_{0}}\right), \beta=\left(r-\frac{\sigma^{2}}{2}\right) \frac{T}{2 m}$ and $\gamma=\sigma^{2} \frac{T}{2 m}$. According to corollary 1 ,

$$
p_{u}=\frac{1}{2}\left(1+\frac{\beta-\alpha}{\sqrt{\gamma}}\left(1+\frac{\alpha(\alpha-2 \beta)}{\gamma}\right)^{-1 / 2}\right)=\frac{1}{2}\left(1+\frac{\beta-\alpha}{\sqrt{\gamma}}+\frac{1}{2} \frac{\alpha(\alpha-\beta)(\alpha-2 \beta)}{\gamma^{3 / 2}}\right)+\mathcal{O}\left(\frac{1}{m^{5 / 2}}\right) .
$$

Next,

$$
\log u=\alpha+\frac{\beta-\alpha}{2\left(p_{u}-\frac{1}{2}\right)}=\alpha+\sqrt{\gamma+\alpha(\alpha-2 \beta)}=\sqrt{\gamma}+\alpha+\frac{1}{2} \frac{\alpha(\alpha-2 \beta)}{\sqrt{\gamma}}+\mathcal{O}\left(\frac{1}{m^{5 / 2}}\right) .
$$

As a consequence,

$$
u=1+\sqrt{\gamma}+\left(\alpha+\frac{\gamma}{2}\right)+\left(\frac{1}{2} \frac{\alpha(\alpha-2 \beta)}{\sqrt{\gamma}}+\alpha \sqrt{\gamma}+\frac{\gamma^{3 / 2}}{6}\right)+\left(\alpha\left(\alpha-\beta+\frac{\gamma}{2}\right)+\frac{\gamma^{2}}{24}\right)+\mathcal{O}\left(\frac{1}{m^{5 / 2}}\right)
$$

and

$2 u p_{u}=1+\left(\frac{\beta-\alpha}{\sqrt{\gamma}}+\sqrt{\gamma}\right)+\left(\beta+\frac{\gamma}{2}\right)+\left(\frac{1}{2} \frac{\alpha(\alpha-\beta)(\alpha-2 \beta)}{\gamma^{3 / 2}}+\sqrt{\gamma} \frac{\alpha+\beta}{2}-\frac{\alpha^{2}}{2 \sqrt{\gamma}}+\frac{\gamma^{3 / 2}}{6}\right)+\mathcal{O}\left(\frac{1}{m^{2}}\right)$.

In the same way, since $\log d=\alpha-\frac{\beta-\alpha}{2\left(p_{u}-\frac{1}{2}\right)}$, one has

$$
d=1-\sqrt{\gamma}+\left(\alpha+\frac{\gamma}{2}\right)-\left(\frac{1}{2} \frac{\alpha(\alpha-2 \beta)}{\sqrt{\gamma}}+\alpha \sqrt{\gamma}+\frac{\gamma^{3 / 2}}{6}\right)+\left(\alpha\left(\alpha-\beta+\frac{\gamma}{2}\right)+\frac{\gamma^{2}}{24}\right)+\mathcal{O}\left(\frac{1}{m^{5 / 2}}\right) .
$$

Next

$$
d+(u-d) p_{u}=1+\left(\beta+\frac{\gamma}{2}\right)+\frac{\gamma}{24}(4 \beta+8 \alpha+\gamma)+\mathcal{O}\left(\frac{1}{m^{5 / 2}}\right)
$$


which ensures that $\left(d+(u-d) p_{u}\right)^{-1}=1-\left(\beta+\frac{\gamma}{2}\right)+\mathcal{O}\left(\frac{1}{m^{2}}\right)$. With $(7)$, one deduces

$\frac{2 u p_{u}}{d+(u-d) p_{u}}=1+\left(\frac{\beta-\alpha}{\sqrt{\gamma}}+\sqrt{\gamma}\right)+\left(\frac{\alpha(2 \beta-\alpha)}{2 \gamma} \times \frac{\beta-\alpha+\gamma}{\sqrt{\gamma}}-\frac{\beta^{2}}{\sqrt{\gamma}}+(\alpha-\beta) \sqrt{\gamma}-\frac{\gamma^{3 / 2}}{3}\right)+\mathcal{O}\left(\frac{1}{m^{2}}\right)$.

From (8), one obtains

$$
\log \left(d+(u-d) p_{u}\right)=\left(\beta+\frac{\gamma}{2}\right)-\left(\frac{\beta^{2}}{2}+\frac{\gamma^{2}}{12}+\frac{\gamma(\beta-\alpha)}{3}\right)+\mathcal{O}\left(\frac{1}{m^{5 / 2}}\right) .
$$

As a consequence

$$
\left(d+(u-d) p_{u}\right)^{-2 m}=e^{-2 m\left(\beta+\frac{\gamma}{2}\right)}\left(1+m\left(\beta^{2}+\frac{\gamma^{2}}{6}+\frac{2 \gamma(\beta-\alpha)}{3}\right)\right)+\mathcal{O}\left(\frac{1}{m^{3 / 2}}\right) .
$$

One easily concludes by replacing $\alpha, \beta$ and $\gamma$ by their expressions in the above expansions.

Proof of Lemma 3 : We follow the approach developped in [4] and first write for $n \in\{2 m, 2 m-1\}$

$$
\sum_{j=0}^{m-1}\left(\begin{array}{l}
n \\
j
\end{array}\right) p^{j}(1-p)^{n-j}=1-\sum_{j=m}^{n}\left(\begin{array}{l}
n \\
j
\end{array}\right) p^{j}(1-p)^{n-j}=1-m\left(\begin{array}{c}
n \\
m
\end{array}\right) \int_{0}^{p} y^{m-1}(1-y)^{n-m} d y .
$$

Making the change of variables $Y=\sqrt{2 m}\left(y-\frac{1}{2}\right)$ in the integral, one easily deduces

$$
\begin{aligned}
& \sum_{j=0}^{m-1}\left(\begin{array}{c}
2 m \\
j
\end{array}\right) p^{j}(1-p)^{2 m-j}=1-2^{1 / 2-2 m} \sqrt{m}\left(\begin{array}{c}
2 m \\
m
\end{array}\right) \int_{-\sqrt{m / 2}}^{\sqrt{2 m}\left(p-\frac{1}{2}\right)}\left(1-\frac{2 Y^{2}}{m}\right)^{m} \frac{d Y}{1+Y \sqrt{\frac{2}{m}}} \\
& \sum_{j=0}^{m-1}\left(\begin{array}{c}
2 m-1 \\
j
\end{array}\right) p^{j}(1-p)^{2 m-1-j}=1-2^{3 / 2-2 m} \sqrt{m}\left(\begin{array}{c}
2 m-1 \\
m
\end{array}\right) \int_{-\sqrt{m / 2}}^{\sqrt{2 m}\left(p-\frac{1}{2}\right)}\left(1-\frac{2 Y^{2}}{m}\right)^{m-1} d Y .
\end{aligned}
$$

Using Stirling formula, $n ! \sim e^{-(n+1)}(n+1)^{1 / 2+n} \sqrt{2 \pi}\left[1+\frac{1}{12 n}+\mathcal{O}\left(\frac{1}{n^{2}}\right)\right]$, one checks that both the coefficients multiplying the integrals in the previous equalities have the following expansion : $\sqrt{\frac{2}{\pi}}\left(1-\frac{1}{8 m}\right)+$ $\mathcal{O}\left(\frac{1}{m^{2}}\right)$.

Then as justified in [4] Theorem 3.4, to obtain the expansion of each integral, one may first expand the integrand in powers of $1 / \sqrt{m}$ and integrate the result term by term. We use Maple to perform these expansions and then to derive the conclusion.

\section{The MSMR method for American Put options}

We have not been able to obtain asymptotic expansions for the price and delta of the American Put Option in the MSM tree with $2 m$ steps and $k=m$. Nevertheless, because of the expansions obtained for the European Put option (see Corollary 2), we propose to use Richardson extrapolation even when computing the price and the delta of the American Put.

For $m \in \mathbb{N}$ such that $2 m \geq\left(\frac{r}{\sigma}-\frac{\sigma}{2}\right)^{2} T$, the $\operatorname{MSMR}(4 \mathrm{~m} / 2 \mathrm{~m})$ method consists in approximating the initial Black-Scholes price and delta respectively by $2 P_{4 m}^{\mathrm{A}}-P_{2 m}^{\mathrm{A}}$ and

$$
2 \frac{v_{4 m}^{\mathrm{A}}\left(1, s_{0} u_{4 m}\right)-v_{4 m}^{\mathrm{A}}\left(1, s_{0} d_{4 m}\right)}{s_{0}\left(u_{4 m}-d_{4 m}\right)}-\frac{v_{2 m}^{\mathrm{A}}\left(1, s_{0} u_{2 m}\right)-v_{2 m}^{\mathrm{A}}\left(1, s_{0} d_{2 m}\right)}{s_{0}\left(u_{2 m}-d_{2 m}\right)} .
$$


where $v_{4 m}^{\mathrm{A}}$ and $v_{2 m}^{\mathrm{A}}$ are computed by the backward induction equations $(3)$ with $\psi(x)=(K-x)^{+}$ respectively in the MSM tree with $4 m$ steps and $k=2 m$ and in the MSM tree with $2 m$ steps and $k=m$.

\section{$5 \quad$ Numerical comparisons}

In this section we compare all the numerical procedures we have mentionned (CRR,BIR,BBSR,AMM), for pricing and hedging plain vanilla American Put options in the Black-Scholes model. We consider four different test cases: A, B, C, D as summarized in Table 3. Each case is associated to a different standard number of steps of the corresponding tree, keeping proper account of the needs of the Richardson extrapolation.

\begin{tabular}{|l|rr|rr|rr|rr|}
\cline { 2 - 9 } \multicolumn{1}{c|}{ CASE } & & A & & B & & C & D \\
\hline & steps & CPU Time & steps & CPU Time & steps & CPU Time & steps & CPU Time \\
\hline CRR & 100 & $\mathbf{1}$ & 200 & 3.80 & 400 & 14.86 & 800 & 59.49 \\
\hline BIR & $50 / 100$ & 1.58 & $100 / 200$ & 5.39 & $200 / 400$ & 19.80 & $400 / 800$ & 76.32 \\
\hline BBSR & $50 / 100$ & 1.97 & $100 / 200$ & 6.10 & $200 / 400$ & 20.85 & $400 / 800$ & 76.73 \\
\hline AMM & $50 / 100$ & 5.43 & $100 / 200$ & 21.48 & $200 / 400$ & 50.95 & $400 / 800$ & 205.95 \\
\hline MSMR & $50 / 100$ & 1.07 & $100 / 200$ & 3.96 & $200 / 400$ & 15.72 & $400 / 800$ & 61.88 \\
\hline
\end{tabular}

Table 3: CPU Times

CPU times will be normalized by the one corresponding to the CRR with 100 time steps. All the computations have been performed in double precision on a PC Pentium $0.8 \mathrm{GHz}$ with $256 \mathrm{Mb}$ of RAM.

\subsection{Sample Selection}

A sample of 5.000 options was extracted randomly from a population whose parameters are similar to the classical ones used in [2] : risk free interest rate $r$ uniform between 0 and 0.1 ; volatility $\sigma$ uniform between 0.1 and 0.6 ; strike $K$ uniform between 70 and 130; time to maturity (years) $T$ uniform between 0 and 1 with probability 0.75 and uniform between 1 and 5 with probability 0.25 ; the initial price of the underlying $s_{0}$ is 100 .

For each option of the sample, the benchmark price is computed in the CRR model with 96.000 steps. In our numerical tests, for each option, the error measure is given by the absolute value of the relative error. Then, the errors for the whole sample are summarized by the Mean Relative Error (MRE) and by the square Root of the Mean Square Relative Error (RMSRE). The values in the tables have been multiplied by $10^{8}$.

Now several options of the sample have been discarded for various reasons. First of all, out of the money options with a very small benchmark price, that is, less than $s_{0} / 1000=0.1$ have been removed. Indeed, such low values are responsible for unreliable and high relative errors. Then, we discarded the in the money options immediately exercisable at the benchmark level, that is, with benchmark price $\left(K-s_{0}\right)$. We signal that this characteristic is correctly perceived for all options of this group by all the pricing methods considered in the test. With this premise these options do not add any pricing error and do not require hedging decisions once they have been correctly exercised. At the end of the story, 4.443 options survived: 2.209 in the money and 2.234 out of the money. 


\subsection{Price Results}

Mean and quadratic errors are presented in Table 4. Notice that for all methods but the CRR tree without Richardson extrapolation, the error is divided by a factor slightly greater than 2 when the step number is multiplied by 2 . In addition, figure 1 offers a speed/precision efficiency graphic in log scale. The curves corresponding to the different methods are approximately straight lines with the same slope. This means that the error behaves as $C /(\text { CPUtime })^{\zeta}$ with $\zeta$ not depending on the method. The smallest multiplicative constant $C$ is obtained with MSMR.

\begin{tabular}{|l|rr|rr|rr|rr|}
\cline { 2 - 9 } \multicolumn{1}{c|}{ PRICE } & & $\mathrm{A}$ & & $\mathrm{B}$ & & $\mathrm{C}$ & & $\mathrm{D}$ \\
\hline 4443 options & MRE & RMSRE & MRE & RMSRE & MRE & RMSRE & MRE & RMSRE \\
\hline CRR & 187873 & 303176 & 93824 & 15053 & 47454 & 76907 & 23332 & 37354 \\
\hline BIR & 14904 & 42027 & 6804 & 29447 & 2647 & 10923 & 982 & 4669 \\
\hline BBSR & 23602 & 60960 & 10240 & 27238 & 4393 & 121570 & 1899 & 5773 \\
\hline AMM & 73471 & 121767 & 37850 & 61987 & 19193 & 31262 & 9764 & 16005 \\
\hline MSMR & 14262 & 40229 & 5795 & 21368 & 2263 & 8122 & 993 & 3174 \\
\hline
\end{tabular}

Table 4: MRE $\times 10^{8}$ and RMSRE $\times 10^{8}$ for the prices of 4443 options.

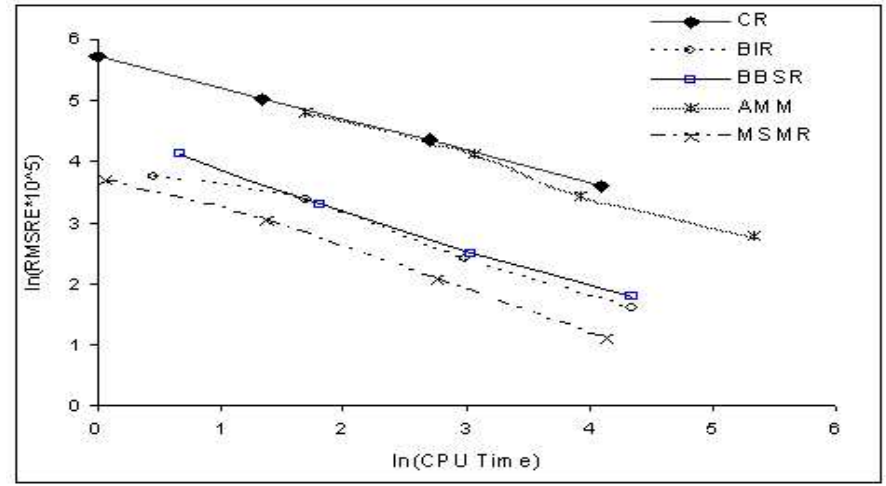

Figure 1: Price: speed-precision efficiency for the 4.443 options.

\subsection{Delta Results}

Mean and quadratic errors are presented in Table 5. In addition, figure 2 offers a speed/precision efficiency graphic in log scale. The behaviour of the different methods is similar to the one observed for the Price. 


\begin{tabular}{|l|rr|rr|rr|rr|}
\cline { 2 - 9 } \multicolumn{1}{c|}{ DELTA } & & $\mathrm{A}$ & & $\mathrm{B}$ & & $\mathrm{C}$ & & $\mathrm{D}$ \\
\hline 4443 options & \multicolumn{1}{c|}{ MRE } & RMSRE & MRE & RMSRE & MRE & RMSRE & MRE & RMSRE \\
\hline CRR & 115467 & 255992 & 57800 & 131320 & 29349 & 70761 & 14540 & 36237 \\
\hline BIR & 45776 & 309916 & 18355 & 127125 & 7612 & 62209 & 3440 & 29834 \\
\hline BBSR & 173104 & 311989 & 88510 & 170322 & 48696 & 107518 & 25788 & 63303 \\
\hline AMM & 74887 & 215847 & 37846 & 106907 & 18319 & 53856 & 9205 & 34411 \\
\hline MSMR & 30161 & 99288 & 13957 & 53971 & 6240 & 30229 & 3773 & 17223 \\
\hline
\end{tabular}

Table 5: MRE $\times 10^{8}$ and RMSRE $\times 10^{8}$ for the deltas of 4443 options.

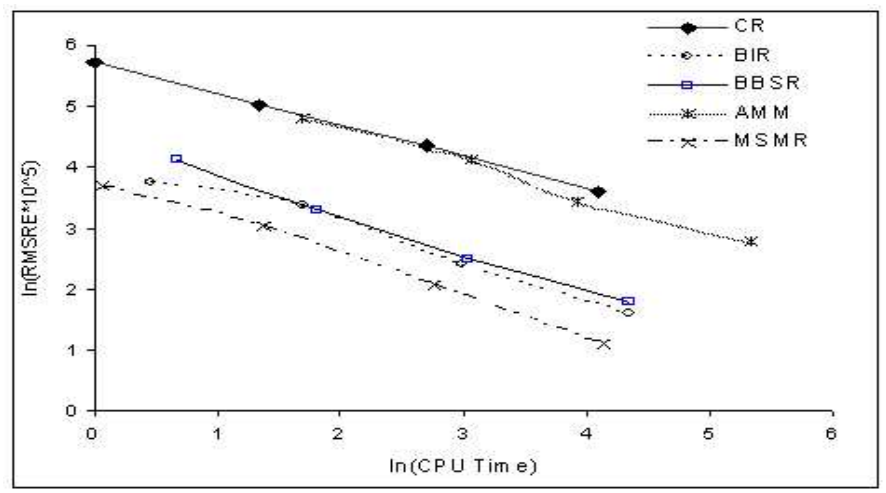

Figure 2: Delta: speed-precision efficiency for the 4.443 options.

\section{Conclusions}

In this paper, we have explicitly constructed MSM binomial trees consistent with the Black-Scholes model in the limit of an infinite step number $N$ and such that the Strike $K$ is equal to the $(k+1)$-th final node of the tree. The most efficient choice of $k$ from a numerical point of view is $k=m$ for $N=2 m$ even. For this choice, we have proved that the difference between the MSM price (resp. delta) and the Black-Scholes price (resp. delta) of the European Put option is given by $\frac{C}{m}+\mathcal{O}\left(\frac{1}{m^{3 / 2}}\right)$ and numerical experiments show that the second term is in fact a $\mathcal{O}\left(\frac{1}{m^{2}}\right)$. This justifies the use of Richardson extrapolation to get rid of the first term in European case. The MSMR method consists in applying the same ideas in the American case. Even if its numerical rate of convergence is only slightly better than $\frac{1}{m}$, it performs better than the other lattice methods as confirmed by an extensive numerical comparison.

Acknowledgments. We are indebted to Laura Ziani for help provided in numerical comparison. We also performed some numerical experiments on the software PREMIA [12].

\section{References}

[1] Black F., Scholes M. : The Pricing of Options and Corporate Liabilities. Journal of Political Economy 81, 637-654 (1973). 
[2] Broadie M., Detemple J. : American Option Valuation: New Bounds, Approximations and a Comparison of Existing Methods. The Review of Financial Studies 9, No. 4, 1221-1250 (1996).

[3] Cox J., Ross S.A. and Rubinstein M. : Option Pricing : A Simplified Approach. Journal of Financial Economics 7, 229-264 (1979).

[4] Diener F. and Diener M. : Asymptotics of the price oscillations of a European Call option in a tree model Mathematical Finance 14, No. 2, 271-293 (2004).

[5] Figlewski S., Gao B. : The Adaptive Mesh Model: a New Approach to Efficient Option Pricing. Journal of Financial Economics 53, 313-351 (1999).

[6] Gaudenzi M. and Pressacco F. : An Efficient Binomial Method for Pricing American Put Options. Decisions in Economics and Finance. Springer Verlag 4, No. 1, 1-17 (2003).

[7] Gaudenzi M., Pressacco F., Zanette A. and Ziani L. : High Precision Pricing and Hedging of American Put Options: New Insights. Working Paper DFIMF. 3, (2004).

[8] Jourdain B. and Martini C. : Approximation of American put prices by European prices via an embedding method. Annals of Applied Probability 12, 196-223 (2002).

[9] Kushner H. Dupuis P.G.: Numerical Methods for Stochastic Control Problems in Continous Time. Springer Verlag (1992).

[10] Lamberton D. : Error estimates for the binomial approximation of American put options. Annals of Applied Probability 8(1), 206-233 (1998).

[11] Lamberton D. : Brownian Optimal Stopping and Random Walks. Applied Mathematics and Optimization 45, 283-324 (2002).

[12] PREMIA : An Option Pricer, Mathfi Project (INRIA, CERMICS, UMLV) http://www-rocq.inria.fr/mathfi/Premia/

[13] Walsh J.B. : The rate of convergence of the binomial tree scheme. Finance and Stochastics 7, 337-361 (2003). 


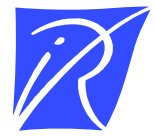

Unité de recherche INRIA Rocquencourt

Domaine de Voluceau - Rocquencourt - BP 105 - 78153 Le Chesnay Cedex (France)

Unité de recherche INRIA Futurs : Parc Club Orsay Université - ZAC des Vignes

4, rue Jacques Monod - 91893 ORSAY Cedex (France)

Unité de recherche INRIA Lorraine : LORIA, Technopôle de Nancy-Brabois - Campus scientifique

615, rue du Jardin Botanique - BP 101 - 54602 Villers-lès-Nancy Cedex (France)

Unité de recherche INRIA Rennes : IRISA, Campus universitaire de Beaulieu - 35042 Rennes Cedex (France)

Unité de recherche INRIA Rhône-Alpes : 655, avenue de l'Europe - 38334 Montbonnot Saint-Ismier (France)

Unité de recherche INRIA Sophia Antipolis : 2004, route des Lucioles - BP 93 - 06902 Sophia Antipolis Cedex (France)

INRIA - Domaine de Voluceau - Rocquencourt, BP 105 - 78153 Le Chesnay Cedex (France)

http://www.inria.fr

ISSN 0249-6399 